die Base leicht in der Kälte auf und lassen sie beim Neutralisieren ausfallen. Konzentrierte Schwefelsäure löst das Carbinol mit roter Farbe, die beim Znsatz eines gleichen Volumens Wasser verschwindet. Diese Farbenreaktion sieht der des Triphenylcarbinols rollkommen gleich.

$0.1588 \mathrm{~g}$ Sbst.: $0.4924 \mathrm{~g} \mathrm{CO}, 0.0823 \mathrm{~g} \mathrm{H} \mathrm{H}_{2} \mathrm{O},-0.1477 \mathrm{~g}$ Sbst.: $0.4585 \mathrm{~g}$ $\mathrm{CO}_{2}, 0.0769 \mathrm{~g} \mathrm{H}_{2} \mathrm{O}$.

$$
\begin{aligned}
& \mathrm{C}_{22} \mathrm{H}_{17} \text { NO. Ber. C 84.83, H 5.50. } \\
& \text { Gef. 》 84.62, 84.71, 》 5.79, 5.79. }
\end{aligned}
$$

Das Hydrochlorid des Carbinols erbält man aus verdünuter salzsaurer Lösung durch Terdunsten im Vakuum. In konzentrierter Salzsäure ist es unlöslich; leicht löslich in Wasser. Aus Alkohol erhält man es in schwach gelb gefärbten Krystallen vom Schmp. 223".

Das Carbinol gibt zwei gut krystallisierende Pikrate; das eine in Alkohol leicht lösliche schmilzt bei $142-144^{\circ}$, während das andere erst bei höherer Temperatur sich schwärzt und zersetzt. Sie enthalten möglicherweise verschiedene Mengen Pikrinsäure.

Das Jodmethylat des Carbinols wurde iiber das Dimethylsulfat dargestellt. Lis stellte einen gelben, krystallinischen Niederschlag dar, der beim Trocknen eine rotbraune Farbe annimmt und um $159^{\circ}$ schmilzt. Das Jodmethylat zeigt eine interessante l'arbenreaktion: Seine Lösung in Wasser gibt mit Natriumcarbonat eine weiße Fällung, die an der Luft bald violett wird. Äther entzieht der alkalischen Flüssigkeit das färbende Prinzip und hinterläßt nach dem Verdunsten einen violetten Körper rom Schmp. $234^{\circ}$.

188. Jakob Meisenheimer:

\title{
Über das Verhalten der Glucose, Fructose und Galaktose gegen verdünnte Natronlauge.
}

[Aus dem Chem. Laboratorium der Landwirtsch. Hochschule gu Berlin.

(Eingegangen am 18. März 1908.)

E. Buchner, J. Meisenheimer und H. Schade ${ }^{1}$ haben etwa vor Jahresfrist gezeigt, dab liructose mit 2-prozentiger Natronlauge bei Gegenwart ron Hydroperoxyd oder unter dem Einflusse des Luftsauerstoffs rasch zerfällt unter Bildung von viel Ameisensäure, wenig Glykolsäure und erheblichen Mengen einer nicht krystallisierenden, mehrwertigen Oxysüure, deren Calciumsalz nach wiederholtem Un-

1) Diese Berichte 39, 4217 [1906].

Berichte d. D. Chem. Gesellschaft. Jahrg. XXXX]. 
fällen mit Alkohol die Eigenschaften und annäluernd auch die $\mathrm{Zu}$ sammensetzung des erythronsauren Calciums besal $\left(\beta^{1}\right)$. Wegen des Zusammenhangs mit den Vorgängen bei der alkoholischen Gürung war es mir von Interesse, das Verhalten der Hexosen gegen verdünnte Natronlauge auch bei Ausschluß eines Oxyclationsmittels quantitativ zu verfolgen, insbesondere um festzustellen, ob, wie nach obigen Versucben ${ }^{2}$ ) zu erwarten war, in diesem Falle neben Milchsïure Erythrousïure als Hauptspaltungsprodukt auftreten würde.

Die in dieser Richtung vorliegenden alteren Versuche ${ }^{3}$ ) erscheinen ebenso wenig entscheidend, wie die neueren von J. U. Nef ${ }^{4}$, da in ibnen eine quantitative Erforschung aller Reaktionsprodukte nicht angestrebt und außerdem unter viel energischeren Bedingungen (höherer Temperatur und größerer Alkalikonzentration) gearbeitet wurde.

Die unten beschriebenen Versuche erstrecken sich auf 3 verschiedene Hexosen, Glucose, Fructose und Galaktose. Glucose und Fructose verhalten sich, wie nach den Arbeiten von Lobry de Bruyn und van Fkenstein über die wechselseitige Umlagerung dieser Zuckerarten in einander unter der Einwirkung von Alkali vorausgesehen werden konnte, recht ähnlich; beide liefern mit verdünnter Natronlauge bei Zimnertemperatur nach monatelangem Stehen über die Hälite ihres Gewichts, 50-60\%, inaktive Milchsäure. Der Rest des Zuckers verwandelt sich der Hauptsache nach, vämlich 30 $-50 \%$ der angewandten. Hexosemenge, in ein Gemisch mehrwertiger Oxysäuren, von dem unten noch weiter die Rede sein

1) Dagegen ist die dort (S. 4230) angegebene weitero Identifizierung dureh Überführung in das Phenylhydrazid, wie sich alsbald (schosı vor Erscheinen der unten zitierten Arbeiten) herausstellte, irrtümlich. Die für $r$-Erythronsäurephenylhydrazid gehaltene Substanz vom Schmp. 126-1280 ist Acetylphenylhydrazin, welches zufälligerweise genau denselben Schmelzpunkt und ähnliche Löslichkeitsverhältnisse, besitzt wie die von $0 . R$ uff (diese Berichte 32, 3680 [1899]; 34, 1369 [1901]) beschriebenen $d$ - und $l$-Erythrousäurephenylhydrazide. Das inzwischen dargestellte wirkliche $r$-Erythronsäurephenylhydrazid selmilyt nach J. U. Nef (Ann. d. Chem. 357, 234 [1907]) bei $151^{\circ}$, nach R. Lespieau (Bull. Soe. Chim. de France [1] 1, 1112 [1907J) bei $145-146^{\circ}$, also viel höher als die Hydrazide der $d$ - und $l$-Säure. Demnach mub es jetzt als recht zweifelhaft gelten, ob die a. a. O. erhaltene Säure wirklich aus reiner oder annähernd reiner Erythronsäure bestand; wahrscheinlich lag ein kompliziertes Säuregemisck ähnlicher Zusammensetzung vor.

2) A. a. O., S. 4224 .

3) Über die umfangreiche Literatur vergl. E. v. Lippmann, Chemie der Zuckerarten (Braunschweig, 1904), S. 328, 713 und 835.

4) Ann. d. Chem. 335, 326 [1904]; 357, 294 [1907]. 
wircl. Die übrigen Produkte treten quantitativ sehr zurïck. Nachgewiesen wurde noch Ameisensäure $(0.5-2 \%)$; ferner entstehen kleine Mengen amorpher, brauner Flocken. Etwa 1\% des Zuckers scheint unter Bildung äjuivalenter Mengen von Kohlendioxyd und Alkohol zu zerfallen. Wegen der vorliegenden geringen Quantität konnte allerdings der letztere nicht mit Sicherbeit als solcher identifiziert werden. Da aber die Substanz mit Wasserdämpeen sehr leicht flüchtig war und starke Jodoformreaktion gab, darf man wohl mit großer Wahrscheinlichkeit auf das Vorhandensein von Alkobol schließen, insbesondere, wenn man die früheren. Versuche von E. Buchner und I. Meisenheimer ${ }^{1}$ ) berücksichtigt. Diese haben unter den \%ersetzungsprodukten von Invertzucker durch heiße, starke Natronlauge ebenfills Alkohol aufgefunden und durch Überführung in den $p$-Nitrohenzoesäureätbylester scharf nachgewiesen.

Es bilden sich sicherlich nicht: Glykolsäure, Oxalsäure, Glykol und Glycerin ${ }^{2}$ ). Auf die beiden mehrwertigen Alkohole wurde besonders sorgfältig geprüft, weil anfänglich das schon erwähnte Gemenge von Polyoxysäuren für ein Gemisch von Glycerin- und Trythronsäure gehalten wurde. Da diese Säuren einen höheren Sauerstoffgehalt als Zucker aufweisen, so hätte die äquivalente Menge eines wasserstoffreicheren Bruchstiicks daneben auftreten miissen. - Außer der schon erwïhnten geringen Nenge Alkohol finden sich in den Reaktionsprodukten keinerlei leichtflüchtige oder durch Kochen mit Jodwasserstofflsäure in niedrig siedende Jodverbindungen iiberïührbare Substanzen, neben Ameisensänre höchstens noch Spuren flüchtiger lettsäuren.

(ralaktose ${ }^{3}$ ) lielert unter den gleichen Bedingungen noch nicht ganz $20 \%$ Milchsäure; dałür gehen etwa $70 \%$ in das Polyoxysäurengemisch ïber. Ameisensäure entsteht ebenfalls in geringer Menge, (ilykol oder Glycerin anch nicht spurenweise.

Was die theoretische Deutung der gefundenen Resultate betrifft, so kann ich mich hier ganz kurz fassen. Über die Milchsäure steht uach den Arbeiten ron (i. Pinkus'), J. U. Nef ${ }^{5}$ ), A. Wohl' ${ }^{6}$ ), A. Windaus und F. Knoop ${ }^{\top}$, sowie E. Buchner, J. Meisenheimer

1) Diese Berichte 38, 624 [1905].

2) Zum gleichen Resultate bezüglich der letzteren beiden Produkte kan auch J. U. Nef, Ann. d. Chem. 335,327 [1904].

3) Vergl. auch A. Windaus, diese Berichte 40, 801 [1907]; J. U. Nef, Anu. d. Chem. 357, 300 [1907].

4) Diese Berichte $\mathbf{3 1}, 31$ [1898].

j) Ann. d. Chem. 335, 254 [1904]]; 357, 296 [1907].

b) In E. v. Lippmanu, Chemie der Zuckerarten (190t), S. 1891.

i) Diese Berichte 38, 1167 [1905]. 
und H. Schade ${ }^{\text {) }}$ wohl soviel mit Sicherheit fest, daß ihrer Bildung der Zerfall des Zuckers in einen Aldehyd oder ein Keton mit dreigliedriger Kohlenstoffkette (Dioxyaceton, Glycerinaldehyd oder Methylglyoxal, vielleicht alle drei neben einander) vorausgeht. Natiirlich kann durch nachträgliche Umlagerung dieser Substanzen durch Alkali nur inaktive Säure entstehen. - Die Ameisensäure dürłte zum Teil auf Oxydation durch Luftsauerstoff infolge ungenügenden Iuftabschlusses, zum Teil wohl aber auch aul direkte Spaltung des Zuckers zurückzuführen sein.

Schwierigkeiten macht die Aufklärung des "Polyoxysäurengemisches«. Nef, der sich in der neuesten Zeit eingeheud mit diesem Teil der Reaktionsprodukte beschäftigt hat, betrachtete ihn zunächst ${ }^{2}$ ) als reine $r$-Erythronsäure, verwirft aber in der letzten Abhandlung ${ }^{3}$ ) diese Ansicht wieder und nimmt nunmehr an, daß das Gemisch ausschließlich aus zahlreichen isomeren, 6 Kohlenstoffatome enthaltenden Saccharinen, $\left.\mathrm{C}_{6} \mathrm{H}_{10} \mathrm{O}_{5}{ }^{4}\right)$, besteht, deren Bildung in theoretisch einleuchtender Weise auseinander gesetzt wird.

Trotzdem muß auch diese neuere Auflassung von Nef, daß bei der Reaktion nur Saccharine mit sechsgliedriger Kette auftreten, unrichtig sein. Es folgt das schon aus seinen eignen, in der ersten Abhandlung ${ }^{5}$ ) angeführten Analysen der erhaltenen Calciumsalze. Jiese ergeben einen Calciumgehalt von $11.85-12.60 \%$, während sich auf saccharinsaures Calcium, $\left(\mathrm{C}_{6} \mathrm{H}_{11} \mathrm{O}_{6}\right)_{2} \mathrm{Ca}$, nur $10.05 \%$ berechnen. Fs müssen also auch Säuren mit geringerer Kohlenstoffatomzahl in dem Gemenge vorhanden sein. Die einfachste Annahme ist die, daß neben den 6 Kohlenstoffatome enthaltenden Saccharinen ähnliche Substanzen mit 4- und 5-gliedrigen Ketten beigemengt sind. Die letzteren müßten einer Spaltung der Kohlenstofikette an verschiedenen Stellen ihre Bildung verdanken, z. B.:

$$
\begin{aligned}
\mathrm{CH}_{2}(\mathrm{OH}) \cdot[\mathrm{CH} . \mathrm{OH}]_{4} \cdot \mathrm{CHO}= & \mathrm{CH}_{2}(\mathrm{OH}) \cdot[\mathrm{CH} \cdot \mathrm{OH}]_{2} . \mathrm{CHO} \\
& +\mathrm{CH}_{2}(\mathrm{OH}) . \mathrm{CHO} .
\end{aligned}
$$

Die so gebildete Erythrose wird in der von $\mathrm{Nef}$ fïr die Hexosen ausführlich entwickelten Weise in $\alpha, \gamma$-Dioxy-buttersäure (die zugehörige "S accharinsäure ") umgewandelt, der Glykolaldehyd wieder aufgebaut und von neuem zersetzt. Frfolgt die obige Spaltung in der Mitte der Kette, so entsteht lediglich Milchsäure.

1) Diese Berichte 39, 4224 [1906]. - Vergl. auch F. Framm, Pflügers Archiv für Physiol. 64, 598 [1896].

2) Ann. d. Chem. 335, 241 [1904]. 3) Ebenda, 357, 215, 294 [1907].

4) Vergl. dazu H. Kiliani, diese Berichte 41, 158, 469 [1908].

5) Ann. d. Chem. 335, 326 [1901]. 
Auch die unten mitgeteilten Analysen des Calciumsalzgemisches aus den Polyoxysäuren sind mit der hier rertretenen Auffassung im Einklange. In einem Falle, bei Verwendung von Galaktose, wurde direkt der Nachweis erbracht, dalis sich das Säuregemisch aus einer Anzahl von Säuren mit verschiedener Kohlenstoffatomzahl zusammensetzt. Bei dem tagelangen lixtrahieren der Milchsüure mit $\ddot{\Lambda}$ ther geht nämlich ein nicht unerheblicher Teil der Polyoxysäuren mit in die ätherische Lösung über. Natürlich sind dies vornehmlich die Säuren mit dem geringeren Molekulargewicht und der kleineren Anzahl von Hydroxylgruppen; tatsächlich stimmt die Calciumbestimmung des daraus gewonnenen Calciumsalzes fast genau für diox ybuttersaures Calcium, während das Calciumsalz des ätherunlöslichen Restes den normalen, niedrigeren Calciumgehalt aufweist. Übrigens zeigen die Kohlenstoffund Wasserstoffzahlen des Calciumsalzes aus Glucose mit liesonderer Schärfe, daß die darin enthaltene Säure die Zusammensetzung $\left(\mathrm{CH}_{2} \mathrm{O}\right)_{\mathbf{x}}$ hat, wobei $\mathrm{x}$ etwa den Wert 5.5 erhält.

Ausgedehntere Versuche zur Trennung des Oxysäuregemisches sind von mir nicht angestellt worden und auch für die \%ukunft nicht beabsichtigt. Bemerkt sei nur, daß die Süuren durch Scbütteln mit Benzoylchlorid und Natronlauge in ein alkaliunlösliches, in Äther schwer lösliches Öl umgewandelt werden. Es geht daraus hervor, daß die Säuren alle $\gamma$-Oxysäuren sind, da die Unlöslichkeit des Benzoylierungsprodulites in Alkali nur durch nebenher laufende Tactonbildung 211 erklären ist. - Durch Einwirkung von Phenylhydrazin krystallisierte Produkte zu isolieren, gelang trotz zahlreicher Versuche nicht.

Die Gegenwart von Natriumsulfit beeinflußt den Verlauf der Zuckerzersetzung durch verdünnte Natroulauge, wie die quantitative Durchführung in 2 lällen zeigt, so gut wie gar nicht; höchstens geht die Reaktion etwas langsamer. Es gelingt, eutgegen den Angaben vou H. Schade'), nicht, auf diese Weise alkalische Zuckerlösungen farblos zu erhalten. Auch ein Zusatz von Cyankalium vermag das nicht; eine Jösung von $2 \mathrm{~g}$ Fructose, $2 \mathrm{~g}$ Cyankalium (= 3 Mol.-Gewichten) und $4 \mathrm{~g}$ Ätzuatron in $250 \mathrm{ccm}$ Wasser färbte sich bereits bimen 24 Stunden dunkelbraunrot. Quantitativ wurde der Versuch nicht durchgefiihrt.

\section{lixperimentelles.}

Bei deu 7 unten beschriebenen Versuchen wurden im großen und ganzen die gleichen Bedingungen eingehalten. Fine beschreibung der allgemein befolgten Versuchsanordnung sei daher der Wiedergabe der Einzelresultate vorangestellt:

1) Ztschr. für physik. Chem. à7, 6 [1906]. 
Eine genau abgewogene Menge Hexose (20-200 g) wurde, erentuell unter Zusatz von Natriumsulfit (5 Mol-Gew.), in der 20-50-fachen Menge $n$-Natronla luge gelöst und in mit Gummistöpsel gut verschlossener, fast bis zum Hals gefüllter Flasche bei limmertemperatur einige Wochen bis Monate im Dunkeln aufbewahrt. Um das Fortschreiten der Zersetzung zu verfolgen, wurde die Lösung von \%eit zu Zeit mit 11/2-Salzsäure unter Anwendung von Phenolphthalein (in selteneren Fïllen Jackmus) titriert. Da die Flüssigkeit stets nach wenigen Tagel dunkelrotbraun gefärbt war, ließen sich die Umschläge nicht mẹhr scharf erkennen; die Unterschiede zwischen der verbrauchten. Menge Ätzuatron und der bei der Aufarbeitung gefundenen Gesamtsäure dïrften zum Teil auf diesen Umstand zurïckzuf̈̈hren seiv. - Bei Unterbrechung des Versuchs wurden stets $10 \mathrm{ccm}$ herauspipettiert und titriert und in der gleichen Probe der noch vorhandene Zucker getwichtsanalytisch nach E. Pfliiger ${ }^{2}$ ) oder Kjeldahl ${ }^{2}$ ) ermittelt.

Die Bestimnung der gebildeten Ameisensäure erfolgte anfïnglich in der Weise, da $35 \mathrm{ccm}$ der neutralisierten lieaktionsfluissigkeit direkt nach A. Lieben') mit Quecksilberchlorid erhitzt wurden. Da in dessen ein Versuch zeigte, daß sich auf diese Weise viel zu hohe Werte ergaben, so wurden späterhin stets $50 \mathrm{ccm}$ unter Zusatz von konzentrierter Phosphorsüure mit Wasserdanp destilliert, das Destillat mit Bariumcarbonat gekocht, die flüchtige Säure nochmals mit Phosphorsäure übergetrieben und erst in diesem Destillat die Ameisensäure durch Reduktion von Quecksilberchlorid bei Gegenwart von Natriumnitrit ${ }^{4}$ ) bestimmt. 1)ie alkalinetrische Titration der flüchtigen Säure lieferte stets höhere Zahlen, als der Imeisensïure allein entsprach, was ohne \%weifel zum Teil auf ein Mitübergeben von Milchsäure, vielleicht aber auch auf das Vorhandensein geringer Mengen anderer niederer lettsäuren zurückzuführen ist. - In den beiden Versuchen, in welchen die \%uckerzersetzung bei Gegenwart von Natriumsulfit vor sich ging, wurde durch das oben erwähnte lirhitzen des ersten Destillats mit Bariumcarbonat gleichzeitig die T'rennung der Ameisensäure von der schwefligen Säure erzielt.

Die entstandene Kohlensüure wurde in der Weise ermittelt, daß $50 \mathrm{ccm}$ in einem geeigneten I pparat mit verdünnter Schwefelsäure erwïrnt und der Gewichtsverlust festgestellt wurde; die Differenz z wischen der so gefundenen und der in der Natronlauge ron vornherein ror-

1) Pflügers Arrhiv für Plıysiol. 93, 163 [1902].

$\Rightarrow$ Ztschr. für analyt. Chem. 35, 348 [1896].

b) Monatsh. für Chem. 14, 753 [1893].

4) E. Buchner, 1. Meisenheimer und H. Schade, diese Berichte 39, 4226 [1906]. 
handenen, auf die gleiche Weise bestiminten Menge ergab den Betrag des nen gebildeten Kohlendioxyds. - Zur Prïfung auf Glykol oder Glycerin wurden in dem Galaktoseversuch $5 \mathrm{~cm}$ der ursprünglichen Lösung mit gesättigter Jodwasserstoffsäure nach S. Zeisel und R. Fanto ${ }^{1}$ ) in dem von M. J. Stritar ${ }^{2}$ ) koustruierten Apparat gekocht; es entstand in der Silbernitrat enthaltenden Vorlage keine Spur einer 'Trübung ${ }^{3}$ ). In anderen Versuchen wurdeu die sämtlichen wäßrigen Destillate (s. u.) nochmals für sich aufs sorgfältigste fraktioniert, um eventuell mit den Wasserdämpfen verflüchtigtes Glykol anfzufinden. Das Thermometer stieg indessen nie über den Siedepunkt des kochenden Wassers, bezw. siedender Salzsäure. Auch mit Benzoylchlorid uud iiberschüssiger Natronlauge entstand kein Niederschlag, während selbstbereitete, wäßrige Glykollösungen bei entsprechender Behaudlung auch noch in starker Verdünnung etwa $80 \%$ der theoretisch berechneten Menge Dibenzoylester in krystallinischer l'orm lieferten.

Der gesamte, nach diesen Vorproben noch ïbrig bleibende Rest wurde mit der berechneten Menge Salzsäure nahezu neutralisiert und zur Hälfte abdestilliert. Mit den Wasserdïmpen ging der Alkohol über, dessen Menge sich im Destillat nach noch mehrmals wiederholtem Übertreiben aus der Erniedrigung des Gefrierpunkts gegenüber den des reinen Wassers ${ }^{4}$ ) ergab. - Im Destillationsrückstand wurden nnnmehr die gesanten organischen Säuren durch Zugabe ron Salzsäure in geringem Überschuß in Freiheit gesetzt, eine geringe Menge aligeschiedener brauner llocken abgesaugt und das Filtrat im Pipschen ${ }^{5}$ ) A pparat erschöpfend mit Äther extrahiert. In den Ätherauszug ging die Milchsäure, welche in Form ihres schwer löslichen Zinksalzes zur Wägung kam. In den Mutterlaugen des Zinklactats fand sich stets ein sehr leicht lösliches, sirupöses Zinksalz, welches in verschiedenen Fällen in das Calciumsalz umgewandelt und einmal als solches auch analysiert wurde. Die Analyse ergab, wie oben bereits erwähnt, einen etwa auf dioxybuttersaures Calcium stimmenden Wert.

Die mit Äther extrahierte Fliissigkeit wurde im Vakuum bei 50 $-70^{0}$ zur Trockne gebracht, sechsmal mit Alkobol ausgezogen, clas

1) Ztschr. für analyt. Chem. 42, 549 [1903].

2) Ztschr. für analyt. Chem. 42, 579 [1903].

3) Im Anschlua an diese Versuche wurde untersucht, ob sich Glykol durch Kochen mit starker Jodwasserstoffsäure quantitativ in Jodäthyl überführen läßt. Es wurden dabei stets riel zu niedrige und wechselnrle Werte gefunclen. Die Ursache dicser überraschenden Tatsache konute nicht anfgeklärt werden.

4) R. Gaunt, Ztschr. für analyt. Chem. 44, 106 [1905].

5) Ztschr. für angew. Chem. 16, 657 [1903]. 
Kt)chsalz abfiltriert und die alkoholische Lösung wiederum in Vakuum vïllig eingedampft. Jie im Rückstande vorhandenen Süuren gaben nach vielstündigem Lrwärmen nit Calciumcarbonat in wäßriger Lösung sehr leicht lösliche Calciumsalze, welche sich nach denı Verdampfen des Wassers durch anhaltendes Verreiben mit Alkohol in eine bräunliche, körnige Masse verwandeln ließen. Diese wurde nach dem Waschen mit absolutem Alkohol und $\ddot{A}$ ther und tagelangem Trocknen im Vakuum über Schwefelsüure gewogen und verschiedentlich auch analysiert. In der alkoholischen Mutterlauge fand sich meist noch etwas unveränderter Zucker.

Bezüglich der nunmehr folgenden Einzelwerte sei noch bemerkt, لlaß im Text die tatsächlich gefundenen analytischen Zahlen wiederregeben sind, während die Tabelle die Gesamtresultate auf $100 \mathrm{~g}$ Zucker umgerechnet enthält.

In den ersten 4 Versuchen ist angenonmen, dab das Calciumsalz die gleiche Zusammensetzung hat wie das analog getrocknete im fünften, d. h. je 10\% Wasser und Calcium enthält; Perner ist in diesen Versuchen die in dem sirupösen Zinksalz vorliegende Menge $\mathrm{Oxy}-$ säuren in der Gesamtaufstellung der aus dem Calciumsalz berechneten hinzugezählt. Die in den ersten Versuchen für Milchsäure gefundenen Werte sind zu niedrig, weil die Ätherextraktion nicht genügend lange (nur 1-2 Tage) fortgesetzt wurde; schließlirh wurde stets 4-5 Tage lang extrahiert. - Was die Bewertung der rerschiedenen Versuchsreiben betrifft, so sind die Ergebnisse der letzten drei Versuche unbedingt als die zuverlüssigeren zu betrachten.

1. Am 12. Dezember 1906 wurden $20.0 \mathrm{~g}$ reiner Traubenzucker in 11 mit Ätzkalk möglichst von Kohlensäure befreiter annähernd $n$-Natronlauge (entsprechend $40.5 \mathrm{~g}$ Ätznatron) gelöst. Gesamtgewicht $1065 \mathrm{~g}$. Die Flüssigkeit färbte sich binnen 3 Stunden dentlich gell. Der ursprüngliche Titer: $5 \mathrm{ccm}(5.22 \mathrm{~g})$ Lösung gleich $9.92 \mathrm{ccm} 1 / 2$-Salzsäure, ging in 2 Tagen auf 9.63 , in 9 Tagen auf $8.80 \mathrm{~cm}$ zurück und betrug am 3. Januar 1907 nocl 8.47, am 8. Januar $8.28 \mathrm{~cm} \mathrm{n} / 2$-Salzsäure. Gleichzeitig enthielten $10 \mathrm{ccm}$ noch

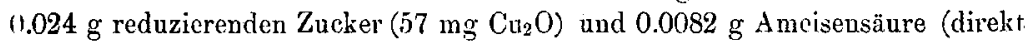
bestimmt, daher sicherlich vicl zu hoch; vergl. unter 2.). Im liest der Lösung $(1018 \mathrm{~g})$ wurden gefunden: $1.05 \mathrm{~g}$ Alkohol $\left(300 \mathrm{ccm}\right.$ Destillat gaben $0.007^{\circ}$ Depr.), $10.0 \mathrm{~g}$ Zinklactat, $1.1 \mathrm{~g}$ nicht krystallisierendes, sirupöses Zinksalz, $8 \mathrm{~g}$ Calciumsalzgemisch und etwa $1.5 \mathrm{~g}$ unlösliche, amorphe Flocken unci sirupöser Rückstand (unzcrstörter Zucker).

2. Genau wie 1. mit $20.0 \mathrm{~g} \mathrm{Frntose} \mathrm{am} \mathrm{gleichen} \mathrm{lage} \mathrm{angesetzt;} \mathrm{dic}$ Lüsung färbte sich binuen wenigen Hinuteu gelb. Während zunäklist $5 \mathrm{~cm}$ $9.83 \mathrm{ccm} n / 2$-Salzsäure entsprachen, waren nach 4 Tagen noch 8.80 , nach 9 Tageu 8.52, nach 22 Tagen 8.17 , nach 27 Tagen 8.07 und nach 35 Tagen, bei Unterbrechung des Versuchs, nur noch $8.00 \mathrm{cem} \mathbf{n}_{2}$-Salzsäure zur Neutralisation erforderlich. Am 8. Januar 1907 enthielten $10 \mathrm{ecm} 0.016 \mathrm{~g}$ reduzierenden Zueker 
(40 $\mathrm{mg} \mathrm{Cu} \mathrm{Cu}_{2} \mathrm{O}$ ), am Tage dex Aufarbeitung $0.009 \mathrm{~g}\left(21 \mathrm{mg} \mathrm{Cu} \mathrm{C}_{2}\right.$ ). Ameisensäure: Im Destillat bestimmt, $0.019 \mathrm{~g}$ in $50 \mathrm{cem}$, während direkt in $10 \mathrm{cem}$ $0.011 \mathrm{~g}$ gefunden wurden. Kohlendioxyd: $0.0338 \mathrm{~g}$ in $50 \mathrm{ecm}$; davon sind $0.0300 \mathrm{~g}$, der Gehalt der ursprünglichen Natronlange in Abzug zu bringen. Alkohol: $0.057 \mathrm{~g}$ in dem Rest $(907 \mathrm{~g}$ ) der noch rorhandenen liösung (46.4 $\mathrm{g}$ Destillat gaben $0.052^{\circ}$ Depr.); in der gleichen Menge: $11.6 \mathrm{~g}$ Zinklactat; 1.6 sirupöses Zinksalz; $7.4 \mathrm{~g}$ Calciumsalzgemisch; $1.2 \mathrm{~g}$ leicht löslicher Riüekstand und Flocken.

3. Genau wie 1. am selben Tage mit $20.0 \mathrm{~g}$ Traubenzucker angesetzt; der Versuch erhielt einen Zusatz von $140 \mathrm{~g}$ neutralem Natriumsulfit. Gesamtgewicht $1205 \mathrm{~g}$. Gelbfärbung trat etwas langsamer als in 1. ein. Zu Beginn entsprachen $5 \mathrm{ccm}(=5.46 \mathrm{~g}) 9.38 \mathrm{~cm}$, nach 24 Stunden 9.27 , nach 9 Tagen 8.34, nach 22 Tagen 8.00 , nach 27 Tagen $7.85 \mathrm{~cm}{ }^{n} / 2$-Salzsäure. Zu dieser Zeit waren in $10 \mathrm{~cm} 0.023$ g Tranbenzucker $\left(55 \mathrm{mg} \mathrm{Cu} \mathrm{Cu}_{2} \mathrm{O}\right.$ ) vorhanden, 13 Tage später (21. Januar 1907; Tag der Unterbrechung) nur noch $0.005 \mathrm{~g}$ (13 $\mathrm{mg} \mathrm{Cu} \mathrm{Cu}_{2} \mathrm{O}$ ); dabei ging der Titer anf $7.73 \mathrm{ccm} \mathrm{n} / 2$-Salzsäure zurück. Ameisensäure, gefunden im Destillat von $50 \mathrm{ccm}: 0.010 \mathrm{~g}$. In den übrig bleibenden $1041 \mathrm{~g}$ wurden bestimmt: $0.06 \mathrm{~g}$ Alkohol (50.4 $\mathrm{g}$ Destillat gaben eine Gefrierpunktserniedrigung ron $\left.0.051^{\circ}\right) ; 11.8 \mathrm{~g}$ Zinklactat; $1.1 \mathrm{~g}$ sirupüses Zinksalz; 9.1 g Calciumsalzgemisch und 0.2 g Rückstand.

Zersetzung von Hexosen durch $n$-Natronlauge.

(Die Zahlen sind auf $100 \mathrm{~g}$ /ucker berechnet.)

\begin{tabular}{|c|c|c|c|c|c|c|c|c|c|c|}
\hline \multirow{3}{*}{ 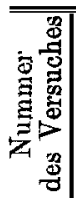 } & \multirow{3}{*}{$\begin{array}{l}\text { Zucker- } \\
\text { art }\end{array}$} & \multirow{3}{*}{ 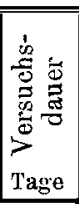 } & \multirow{3}{*}{\multicolumn{2}{|c|}{\begin{tabular}{c|c}
$\begin{array}{c}\text { Ange- } \\
\text { wandtes }\end{array}$ & $\begin{array}{c}\text { Ver- } \\
\text { brauchtes }\end{array}$ \\
$\mathrm{NaOH}$ in \\
(bezw. Mol.-Gew.)
\end{tabular}}} & \multirow{3}{*}{ 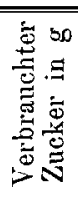 } & \multicolumn{3}{|c|}{ Reaktiousprodukte } & \multirow{2}{*}{\multicolumn{2}{|c|}{$\begin{array}{l}\text { Aufgeklärter } \\
\text { Verbrauch an }\end{array}$}} \\
\hline & & & & & & \multirow{2}{*}{$\begin{array}{l}\text { Ameisen- } \\
\text { sïure }\end{array}$} & \multirow{2}{*}{$\begin{array}{l}\text { Milch- } \\
\text { säure }\end{array}$} & \multirow{2}{*}{$\begin{array}{l}\text { Poly- } \\
\text { oxy- } \\
\text { säuren }\end{array}$} & & \\
\hline & & & & & & & & & Zucker & $\mathrm{NaOH}$ \\
\hline 1 & Glucose & 27 & $202.5(9.1)$ & $33.5(1.5)$ & 87.5 & $\left.4.2^{1}\right)$ & 31.5 & 40 & 74 & 28.0 \\
\hline 2 & Fruc & 35 & 202 & $37.7(1.7)$ & 95.4 & 2.0 & 41 & 42 & 85 & 31.0 \\
\hline 3 & Glucose $^{2}$ ) & 40 & $202.5(9.1)$ & $33.0(1.5)$ & 97.1 & 1 . & 41 & 47 & 8 & 31.0 \\
\hline 4 & Fructose $\left.{ }^{2}\right)$ & 40 & $202.5(9.1)$ & $34.5(1.6)$ & 97.3 & 0 . & 50 & 4 & 9 & 34.5 \\
\hline 5 & Glucose & 91 & $89.0(4)$ & $37.6(1.7)$ & 98.4 & 0.5 & 53.0 & 37 & 90 & 33.0 \\
\hline 6 & Fructose & 139 & $89.0(4)$ & $34.2(1.5)$ & 100.0 & 0.5 & 54.0 & 36 & 90 & 33.5 \\
\hline 7 & Galaktose & 188 & $89.0(4)$ & $29.1(1.3)$ & 100.0 & 1.5 & 17.8 & 69 & 89 & 26.5 \\
\hline
\end{tabular}

4. Wie 3. mit $20.0 \mathrm{~g} \mathrm{Fructose} \mathrm{ebenfalls} \mathrm{am} \mathrm{12.} \mathrm{Dezember} 1906$ angesetzt. Gesamtgewicht infolge Verwendung einer etwas geringeren Menge Wasser $1165 \mathrm{~g}$. Die Färbung erfolgte auch hier dentlich langsamer als im Parallelversuch 2. $5 \mathrm{ccm}(=5.5 \mathrm{~g})$ neutralisierten zunïchst $9.62 \mathrm{ccm}$, nach 9 Tagen 8.44, nach 22 Tagen 8.20 , nach 27 Tagen noch $8.07 \mathrm{ccm} \mathbf{n}_{/ 2}-$ Salzsäure. Noch vorhandener Zucker zu diesem Zeitpunkte: $0.016 \mathrm{~g}$ in $10 \mathrm{~cm}(40 \mathrm{mg}$ $\left.\mathrm{Cu}_{2} \mathrm{O}\right)$, am 21. Januar 1907 noch $0.005 \mathrm{~g}\left(13 \mathrm{mg} \mathrm{Cu_{2 }} \mathrm{O}\right)$; liter $7.98 \mathrm{~cm}$

1) $\mathrm{Zu}$ hoch bestimmt; vergl. Versuch 1 ,im T'ext.

2) Unter Zusaty von $140 \mathrm{~g} \mathrm{Na} 2 \mathrm{SO}_{3}+7 \mathrm{H}_{2} \mathrm{O}$ (5 Mol.-Gew.). 
n/2-Salzsäure. Ameisensäure $0.007 \mathrm{~g}$ in $50 \mathrm{cem}$. A us den restierenden $1055 \mathrm{~g}$ Flüssigkeit wurden isoliert $15.1 \mathrm{~g}$ Zinklactat, $0.9 \mathrm{~g}$ sirupöses Zinksalz, $9.5 \mathrm{~g}$ Calciumsalzgemisch und $0.3 \mathrm{~g}$ Rückstand.

Die gesanmelten Zinksalze von 1.-4. wurden einmal aus Wasser amkrystallisiert; erhalten $40 \mathrm{~g}$ lufttrockner; schwach rötlich gefärbter Krystalle. Davon verloren $2.0243 \mathrm{~g}$ bei $105^{\circ} 0.3664 \mathrm{~g}$ an Gewicht uncl hinterlielen buim Verglühen $0.5577 \mathrm{~g} \mathrm{ZnO}$.

$$
\begin{aligned}
\left(\mathrm{C}_{3} \mathrm{H}_{5} \mathrm{O}_{3}\right)_{2} \mathrm{Zn}+3 \mathrm{H}_{2} \mathrm{O} . & \text { Ber. } \mathrm{H}_{2} \mathrm{O} 18.16, \mathrm{Zn} 21.99 . \\
& \text { Gef. » } 18.10, \text { »2.14. }
\end{aligned}
$$

Zweite Krystallisation: $3.3 \mathrm{~g}$. Davon gaben $1.0106 \mathrm{~g}$ bei $105^{\circ} 0.1794 \mathrm{~g}$ $\mathrm{H}_{2} \mathrm{O}$ ab und hinterliehen $0.2866 \mathrm{~g} \mathrm{ZnO}$. Gef.: $\mathrm{H}_{2} \mathrm{O} 17.75, \mathrm{Zn} 22.76 \%$.

Die Mutterlauge davon wurcle mit Schwefelwasserstoff gefällt, in das Kupfersal\% verwandelt und auf $1-2 \mathrm{cem}$ eingedampft. Nach tagelangem Stehen krystallisierte 1 g blabblauer Nadeln.

$0.6370 \mathrm{~g}$ Sbst. (lufttrocken): $0.0692 \mathrm{~g} \mathrm{H}_{2} \mathrm{O}, 0.1736 \mathrm{~g} \mathrm{CnO}$.

$$
\begin{array}{lcccc}
\left(\mathrm{C}_{3} \mathrm{H}_{5} \mathrm{O}_{3}\right)_{2} \mathrm{Cu}+2 \mathrm{H}_{2} \mathrm{O} . & \text { Ber. } \mathrm{H}_{2} \mathrm{O} & 12.97, \mathrm{Cn} & 22.91 . \\
\left(\mathrm{C}_{2} \mathrm{H}_{3} \mathrm{O}_{3}\right)_{2} \mathrm{Cu} . & \gg & \text { } & -, & 29.82 . \\
& \text { Gef. } & \text { \% } & 10.86, & 21.77 .
\end{array}
$$

Auch diese letzte Krystallisation bestand also noch aus ziemlich reinem, milchsaurem Kupfer ohne wesentliche Beimengung von glykulsaurcm Salz.

5. $200 \mathrm{~g}$ Glucose am 5. Februar 1907 in $3960 \mathrm{~cm}$ Natronlauge (enthaltend $178 \mathrm{~g}$ Ätznatron $=4$ Mol.-Gew.) gelöst. Gesamtgewicht $4350 \mathrm{~g}$. $10 \mathrm{cem}=10.52 \mathrm{~g}$ erforderten zur Neutralisation $21.8 \mathrm{ccm}$, am SchluB des Versuchs (7. Mai 1907) $12.6 \mathrm{ccm}$ n/2-Salzsüure und enthielten noch $0.008 \mathrm{~g}$

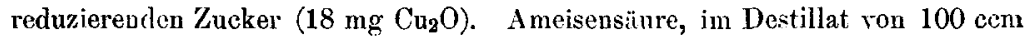
bestimmt: $0.024 \mathrm{~g}$. Beim Ansäneru des zur weiteren Verarbeitung gelangenden Restes $(4215 \mathrm{~g}$ ) schieden sich etwa $0.5-1 \mathrm{~g}$ brauner Flocken ab. Der in Äther gelöste Anteil lieferte neben $1-2 \mathrm{~g}$ in Wasser unlislichen Öles $170 \mathrm{~g}$ Zinklactat und $13 \mathrm{~g}$ Calciumsalz der Polyoxysäuren; letzteres wurde mit der aus der sauren, wäBrigen Lösung gewonnenen Menge vereinigt und so nach tagelangem 'Trocknen in Vakuum über Schwefclsäure $\$ 9 \mathrm{~g}$ Calciunsalzgemisch erhaltrn.

$1.4690 \mathrm{~g}$ Sbst. verloren bei $105^{\circ} 0.1432 \mathrm{~g}(9.75 \%)$ an Gewicht und gaben $0.5006 \mathrm{~g} \mathrm{CaSO}$ (entsprechend $10.03 \% \mathrm{Ca}$ anf die wasserhaltige oder $11.10^{\circ} \%$ auf die trockne Substanz berechnet).

$0.1430 \mathrm{~g}$ Sbst. (Wasserfrei): $0.1885 \mathrm{~g} \mathrm{CO}, 0.0714 \mathrm{~g} \mathrm{H}_{2} \mathrm{O} .-0.1727 \mathrm{~g}$

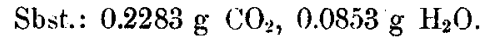

Berechnet man die Kohlenstoff- und Wasserstoff\%ahlen auf aschefroie Substanz unter Berücksichtigung der Tatsache, daß der freien Süure tin Zwanzigstel rom Gewicht des gefundenen Calciums als Wasserstofe zugezählt werden nuB, so ergibt sich Folgendes:

$$
\begin{array}{ll}
\left(\mathrm{CH}_{2} \mathrm{O}\right)_{x} . & \text { Ber. C } 40.0, \quad \text { H } 6.7 . \\
& \text { Gef. " } 40.2,40.3, " 6.8,6.7 .
\end{array}
$$

Als leicht löslicher Rückstand des Caleiumsalzes blieben etwa $2 g$ Sirup. 
6. Am gleichen Tage wie 5. mit $100 \mathrm{~g}$ Fractose und 21 Natronlange (mit einem Gehalt von $89 \mathrm{~g}$ Atznatron) angesetzt. Gewicht $2200 \mathrm{~g} .10 \mathrm{ccm}$ $=10.5 \mathrm{~g}$ neutralisierten zu Beginn 21.3, am Sehluls (24. Juni 1907) $13.1 \mathrm{ccm}$ " $/ 2$-Salzsäure. Sämtlicher Zucker war bis auf Spuren verbraucht. Ameisensïure in $100 \mathrm{ccm}: 0.025 \mathrm{~g}$. Der verbleibende Rest $(2075 \mathrm{~g})$ wurde nahezu neutralisiert und anf etwa $1 \mathrm{l}$ eingeengt. Als nunmehr 1 'lag mit Äther extrahiert wurde, ging so gut wie nichts in den Äther (kein Glykol!). Weiterhin gefunden: $84 \mathrm{~g}$ Zinklactat; $6 \mathrm{~g}$ Calciumsalz aus der Ätherlïsung und $34 \mathrm{~g}$ aus dem wäbrigen Teil, beides nach worhenlangem Tracknen im Vakuum gewogen und nach dem Vermischen analysiert:

$0.8735 \mathrm{~g}$ Sbst, verloren bei $105^{\circ} 0.0436 \mathrm{~g}$ an Gewiclut und lieferten $0.3176 \mathrm{~g} \mathrm{CaSO}{ }_{4}$. Gofunden: $5.0 \%$ Gewichtsverlust, $10.07 \% \mathrm{Ca}(11.25 \%$ wasserfrei).

Flocken und leicht löslicher liückstand etwa $1 \mathrm{~g}$.

7. Genau wie 6. mit $100 \mathrm{~g}$ Galaktose. Der ursprüngliche Titer von $21.4 \mathrm{ccm}$ ging bis zum 12. Juli $1907 \mathrm{nur}$ auf $14.4 \mathrm{ccm}$ n/2-Salzsäure zurück, obwohl auch hier aller Zucker bis auf Spuren zerstört war. Die Färbung blieb tief dnnkelrot. A meisensăure: $0.070 \mathrm{~g}$ in $100 \mathrm{cem}$. Es restierten $2050 \mathrm{~g}$. Daraus isoliert: $27 \mathrm{~g}$ Zinklactat; $12.5 \mathrm{~g}$ Calciumsal\% aus der Ätherlösung (3 Monate in Vakunm getrocknet):

$0.9868 \mathrm{~g}$ Shst. galuen $0.0367 \mathrm{~g}$ (rewiclitsverlust bei $105^{\circ}$ und $0.4806 \mathrm{~g}$ $\mathrm{CaSO}_{4}$; also gefunden: $3.7 \%$ Gewiclitsverlust und $14.35 \%$ Ca $(14.90 \%$ wasserfrei). Dioxybuttersaures Calcium, $\left(\mathrm{C}_{4} \mathrm{H}_{7} \mathrm{O}_{4}\right)_{2} \mathrm{Ca}$ : Ber. Ca 14.4.

Aus der salzsauren Flüssigkeit wurden 63 g ebenso getrocknetes Calciumsalzgemisch gewonnen.

$1.2506 \mathrm{~g}$ Sbst. verloren bei $105^{\circ} 0.0445 \mathrm{~g}$ an Gewicht und gaben $0.4649 \mathrm{~g}$ Ca $\mathrm{SO}_{4}$; also: $3.6 \%$ Gewichtsverlust, $10.96 \% \mathrm{Ca}$ (11.36\% wasserfrei).

Flocken und sirupöser Rückstand etwa $1 \mathrm{~g}$.

\section{Emil Fischer: Reduktion des Glykokollesters ${ }^{1}$ ).}

[Aus dem Chemischen Institut der Universität Berlin.]

(Eingegangen am 16. März 1908.)

Im Gegensatz zu den Estern der gewöhnlichen aliphatischen oder aromatischen Säuren wird der Oxalester bekanntlich durch Natriunamalgam leicht reduziert und die hierbei entstehenden Produkte sind noch kürzlich von W. Traube ${ }^{2}$ ) ausführlich untersucht worden.

Diese Sonderstellung verdankt er offenbar der unmittelbaren Verkettung der beiden stark negativen Carbäthoxylgruppen. Eine ähn-

1) Bei Gelegenheit des Vortrags, den Hr. C. Neuberg in der Sitzung vom 24. Februar d. J. über Aminoaldehyde hielt, habe ich bereits erwähnt, daß ich mit ähnlichen Versuchen beschäftigt sei.

$\Rightarrow$ Diese Berichte 40, 4942 [1907]. 